\title{
Estimating 3D lumen centerlines of carotid arteries in free-hand acquisition ultrasound
}

\author{
Diego D. B. Carvalho - Stefan Klein - Zeynettin Akkus • \\ Gerrit L. ten Kate • Arend F. L. Schinkel • Johan G. Bosch • \\ Aad van der Lugt • Wiro J. Niessen
}

Received: 8 January 2011 / Accepted: 6 June 2011 / Published online: 29 June 2011

(C) CARS 2011

\begin{abstract}
Purpose The purpose of this paper is to present a methodology to estimate the carotid artery lumen centerlines in ultrasound (US) images obtained in a free-hand examination. Challenging aspects here are speckle noise in US images, artifacts, and the lack of contrast in the direction orthogonal to the US beam direction.

Method An algorithm based on a rough lumen segmentation obtained by robust ellipse fitting was developed to deal with these conditions and estimate the lumen center in 2D B-mode scans. In a free-hand sweep examination, continuous image acquisitions are performed through time when the radiologist moves the probe on the patient's neck. The result is a
\end{abstract}

D. D. B. Carvalho $(\varangle) \cdot$ S. Klein · W. J. Niessen

Department of Radiology and Med. Informatics,

Biomedical Imaging Group Rotterdam, Erasmus MC,

Rotterdam, The Netherlands

e-mail: d.carvalho@erasmusmc.nl

Z. Akkus · J. G. Bosch

Biomedical Engineering, Thoraxcenter, Erasmus MC,

Rotterdam, The Netherlands

G. L. ten Kate · A. F. L. Schinkel

Department of Internal Medicine, Division of Pharmacology,

Vascular and Metabolic Diseases, Erasmus MC,

Rotterdam, The Netherlands

A. F. L. Schinkel

Department of Cardiology, Thoraxcenter, Erasmus MC,

Rotterdam, The Netherlands

A. van der Lugt

Department of Radiology, Erasmus MC, Rotterdam,

The Netherlands

W. J. Niessen

Imaging Science and Technology, Faculty of Applied Sciences,

Delft University of Technology, Delft, The Netherlands series of images that show 2D cross-sections of the carotid's morphology. A tracking sensor (Flock of Birds) was attached to the probe and both were connected to a PC executing the Stradwin software, which relates spatial information to the acquisition data of the US probe. The spatial information was combined with the $2 \mathrm{D}$ lumen center estimates to provide a centerline in 3D. For validation, 19 carotid scans from 15 different patients were scanned, their centerlines calculated by the algorithm and compared with results acquired by manual annotations.

Results The average Euclidean distance between both among all the examinations was $0.82 \mathrm{~mm}$. For each examination, the percentage of these Euclidean distances below $2 \mathrm{~mm}$ was calculated; the average over all examinations was $92 \%$.

Conclusion Automated 3D estimation of carotid artery lumen centerlines in free-hand real-time ultrasound is feasible and can be performed with high accuracy. The algorithm is robust enough to keep the centerlines inside the vessel, even in the absence of contrast in parts of the vessel wall.

Keywords Free-hand ultrasound - Atherosclerosis · Carotid lumen centerline

\section{Introduction}

Medical imaging studies of the carotid artery generally aim to observe the presence of atherosclerotic plaque and the effect on the geometry of the vessel lumen. Both atherosclerotic plaque, as well as luminal stenosis are related to cerebrovascular diseases [1]. Several imaging modalities are used, such as magnetic resonance imaging (MRI), computed tomography angiography (CTA), and ultrasound (US), each having their own advantages and disadvantages. MRI has good soft-tissue contrast allowing plaque composition 
analysis in 3D, but the resolution of the images is often limited, especially in the slice direction. CTA visualizes the 3D lumen geometry with high resolution and clearly shows calcifications, but other plaque components (lipids, fibrous tissue, hemorrhage) are hard to distinguish. US provides 2D images with high in-plane resolution allowing intima-media thickness measurements, it has high temporal resolution enabling motion analysis to measure the distensibility of the artery, but may present speckles, noise and a lack of contrast in the direction perpendicular to the beam direction. A good scenario could be to combine the information from MRI, CTA, and US by an integrated analysis, in order to extract plaque characteristics that cannot be identified using a single modality. For such a multimodal analysis, co-registration of the different modalities would be helpful. There are several issues that make the registration of US with MRI/CTA challenging. Firstly, the US scans are mostly acquired in a 2D mode; slices are acquired instead of volumes. Secondly, the imaging characteristics are completely different, which complicates the use of purely intensity-based registration methods (for example, using mutual information). Thirdly, the initial alignment between MRI/CTA and US is rather arbitrary (it depends on the scanner's coordinate system and the position of the US probe). The lumen centerline seems a good candidate for a geometrical landmark that can be extracted reliably in each modality, based on which a robust initial alignment procedure can be developed.

In this work, we present a method to track 3D lumen centerlines of the common, internal, and external carotid artery, from 2D B-mode US scans obtained in a free-hand acquisition, where the probe is gradually moved along the neck of the patient, imaging transverse sections. Each individual ultrasound image in this 2D free-hand acquisition represents a plane cut of a volumetric area (in our case, a region of the patient's neck), but with unknown information about the plane orientation and its position in a three-dimensional space. An attached tracking device is necessary to record the probe's position and orientation during the acquisition. There are also 3D ultrasound techniques, in which 2D images are acquired from a known position through mechanical displacement of the probe [2]. However, 2D probes are more widely used, because they have a higher temporal resolution. Intima-media thickness (IMT) is usually measured in longitudinal sections of the carotid [3]. The observation of the carotid from that perspective also allows to quantify the extension of the atherosclerotic plaque and to analyze the behavior of the wall's motion, but it does not provide enough data to build a good three-dimensional representation of the vessel.

The extraction of centerlines from tubular structures in other modalities, than free-hand ultrasound, has been addressed in different works [4-9]. Schaap et al. [10] give an extensive survey of the literature and present an experimental comparison of algorithms to extract centerlines of coronary arteries. Hameeteman et al. [11] performed a comparison of different algorithms to extract carotid arteries in CTA. Noble and Boukeroui [12] present a survey of different techniques for the segmentation of B-mode US images, including vessels.

The publications most related to our research are from Abolmaesumi and Sirouspour [13], Golemati et al. [14], Hammer et al. [15], and Wang et al. [16,17]. Abolmaesumi \& Sirouspour developed a technique to extract boundaries for cavities in ultrasound images. Their method identifies the boundaries by tracing equispaced radii from a given seed point that lies inside the cavity; visually well-defined borders are assumed. Golemati et al. employed the Hough transform to segment arterial sections during wall motion analysis of the carotid in transverse and longitudinal sections. The difficulty in applying this technique on a free-hand sweep lies in choosing the appropriate parameters. Due to the probe position and the anatomy of the carotid artery, the artery can be represented on the images in elliptical shapes of different radii across the same series. Hammer et al. presented a methodology to build 3D representations of the carotid and femoral arteries from free-hand US acquisitions (using a tracking device) by semi-automated vessel segmentation. Wang et al. developed an algorithm to track the area of the CCA and jugular vein in transversal sections. In their technique, the ray casting method is employed to identify the vessels boundaries and an ellipse is fitted using the end points of the rays. In other to achieve a better adjusted ellipse, there is an intermediate step in which outliers rays are pruned before the fitting.

In this paper, an algorithm inspired by Wang's Spoke Ellipse algorithm $[16,17]$ is proposed to semi-automate the calculation of centroids of the common, internal, and external carotid in each 2D scan. Our method requires only three manually placed seed points, indicating the common (CCA), internal (ICA), and external (ECA) carotid arteries. The algorithm is designed to be robust against missing edge information in parts of the carotid wall, due to the lack of contrast in directions orthogonal to the US beam direction. A magnetic tracking device called Flock of Birds (FOB) (Ascension Technology, Burlington, VT, USA) was attached to the US probe, to register the displacements during the acquisitions. To synchronize the sensor information with the images, we used the publicly available Stradwin software [18]. The next section presents the algorithm for the centerline extraction.

\section{Method}

To estimate a 3D lumen centerline of the carotid arteries, based on 2D transversal free-hand US acquisitions, the lumen centroid must be identified in each 2D image. Using the data from the tracking device, the centroids can be expressed in 3D 


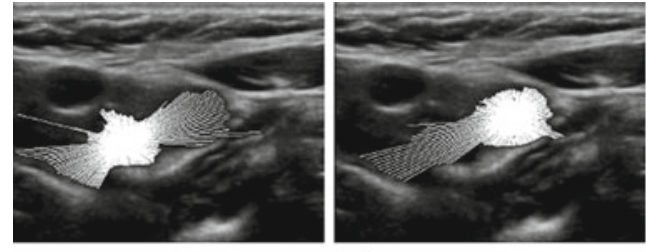

step 1

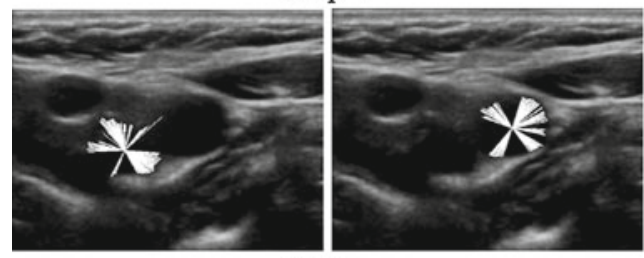

step 2

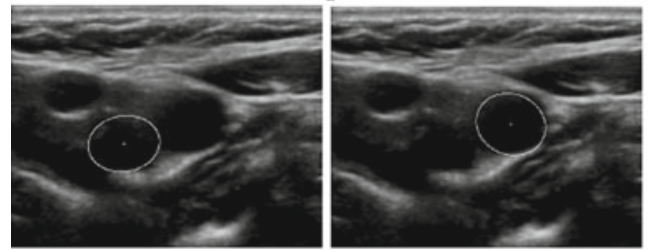

step 3

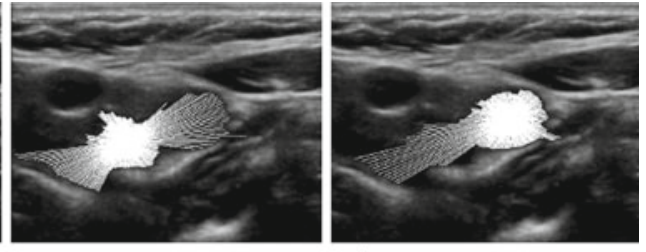

step 4

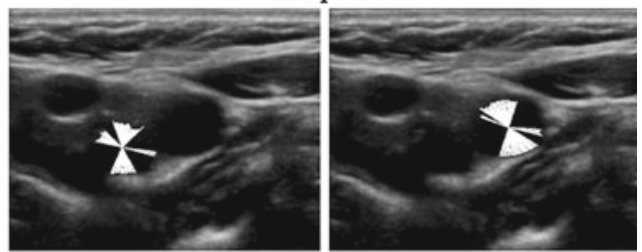

step 5

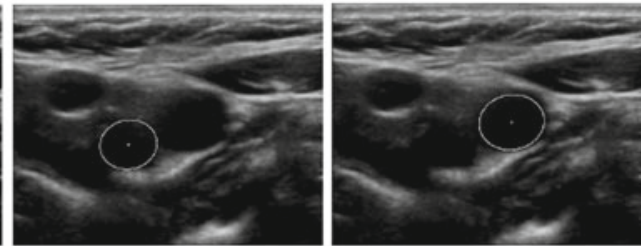

step 6

Fig. 1 Images registering the outputs of each one of the six steps of the algorithm for both ICA and ECA in the same slice

coordinates, which allows construction of a smooth 3D center line.

In 2D B-mode US images, the carotid's lumen is represented by a relatively low intensity, and the vessel wall has a higher intensity. In transverse sections, the vessel wall is visible as an (approximately) ellipsoid structure. Part of the vessel wall is often missing though (see for example Fig. 1), due to the lack of contrast in the direction perpendicular to the US beam direction. We developed a robust ellipse fitting algorithm that can deal with this situation.

The algorithm is composed of 6 steps, which are executed on each US 2D image. The output of each step is illustrated in Fig. 1.

Step 1: The algorithm starts by tracing rays in many directions from a seed point (Point $O$ in Fig. 2) that is known to be inside the vessel. The angle between two rays is defined by dividing $360^{\circ}$ by the number of rays that will be traced. In our experiment, we used 256 rays. The length of each ray is limited by the position where it reaches a pixel with intensity above a certain threshold $(\tau)$, indicating that the vessel wall is being crossed (Point $P$ ).

Step 2: Since we expect the vessel's cross-section to have a more or less circular shape, we expect a similar length among the rays. Outliers are indicative of missing edges (causing very long rays) or noise within the lumen (causing very short rays). In order to discard the outlier rays, a given percentage $(\rho)$ of the rays that have smallest length and the same

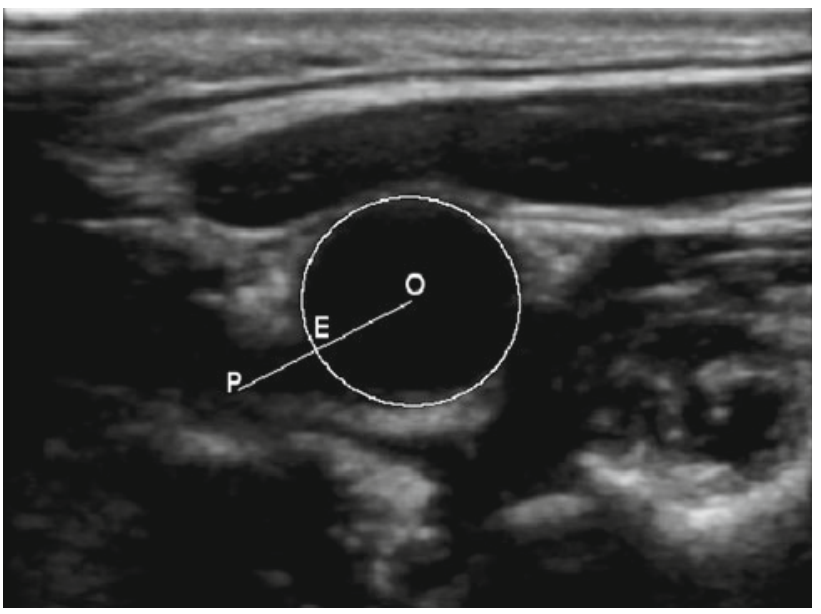

Fig. 2 Points $O, E$ and $P$

percentage of the rays that have longest length are pruned.

Step 3: An ellipse is fitted to the extreme points of each remaining ray. The method presented in the OpenCV library is used to fit the ellipse [19].

Step 4: A new set of rays is traced using the center of the ellipse as the initial point (Point $O$ ).

Step 5: A second fine-pruning step is performed. For each ray, the distance is calculated between the end Point $P$ and the point where it has intersected the previously fitted ellipse (Point $E$ ). The standard deviation and mean of the $E P$ length are calculated. If for a given ray, the length of the line segment $O P$ 

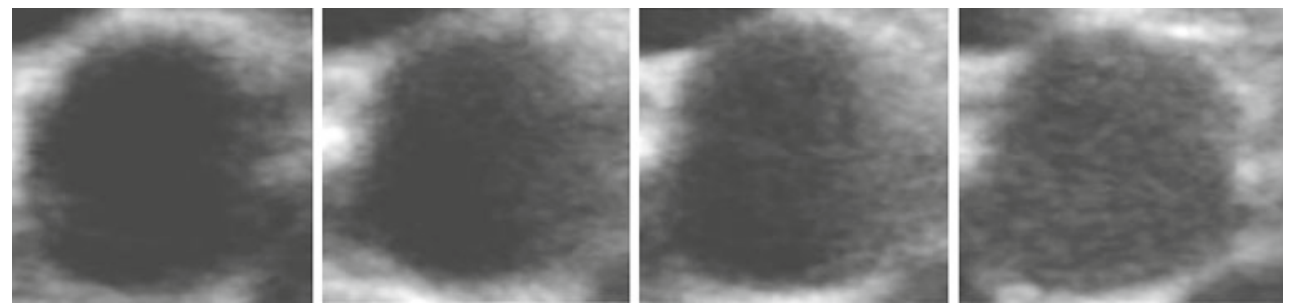

Fig. 3 Four US images of the same patient, at different positions along the CCA. The speckle characteristics vary from slice to slice

is bigger than the length of $O E$ and its $E P$ length is one standard deviation away from the mean of other EP lengths, the Point $P$ is disconsidered and Point $E$ is used. If $\mathrm{OE}$ is larger than OP, the ray is always preserved. By discarding only points that are outside the ellipse, we introduce a bias to shorten rays to increase the chance that the centerpoint remains within the lumen in cases where large points of the lumen boundary are missing. This step is necessary in cases where the edge information in a large part of the lumen boundary is missing.

Step 6: By the last, the remaining rays are used to fit a second ellipse which the centerpoint is saved.

This centerpoint is used as the seed point in the next image in the series and then these 6 steps are repeated. We are initializing the algorithm with three seed points, one at each extreme of the carotid. The algorithm starts at the points in the internal and external carotid and traverses through the scans while calculating both centroids, until they are $1 \mathrm{~mm}$ away, at which point the bifurcation is assumed to be located. Then the algorithm starts in the seed of the common carotid and stops when it reaches the slice where the seed points of the bifurcation were located. This approach is invariant to the direction (from the patient's shoulders to head, or viceversa) that the free-hand acquisition was performed alongside the neck. In the end, the resulting centerpoints are smoothed (Gaussian kernel with standard deviation of $1 \mathrm{~mm}$ ) and interpolated by three spline curves, using 3rd order polynomial splines: one for CCA, one for the ICA, and one for ECA.

Since the lumen and vessel wall intensity distributions may vary between images (even of the same series), we developed a procedure to determine $\tau$ based on the intensity distribution around the three initial seed points. Before starting tracing rays, a kernel of size $1 \times 1 \mathrm{~mm}^{2}$ is created around each of the three seed points. The mean $(\mu)$ and the standard deviation $(\sigma)$ of the pixel intensities in the kernel are calculated. The threshold value is then set to $\tau=\mu+2.5 \sigma$, assuming a Gaussian distribution of the lumen intensities. A user defined minimum standard deviation $\left(\sigma_{\min }\right)$ is enforced to assure that even if the kernel is located in an area with no variation in pixel intensities, the threshold will have a higher value than the pixel at the seed point.
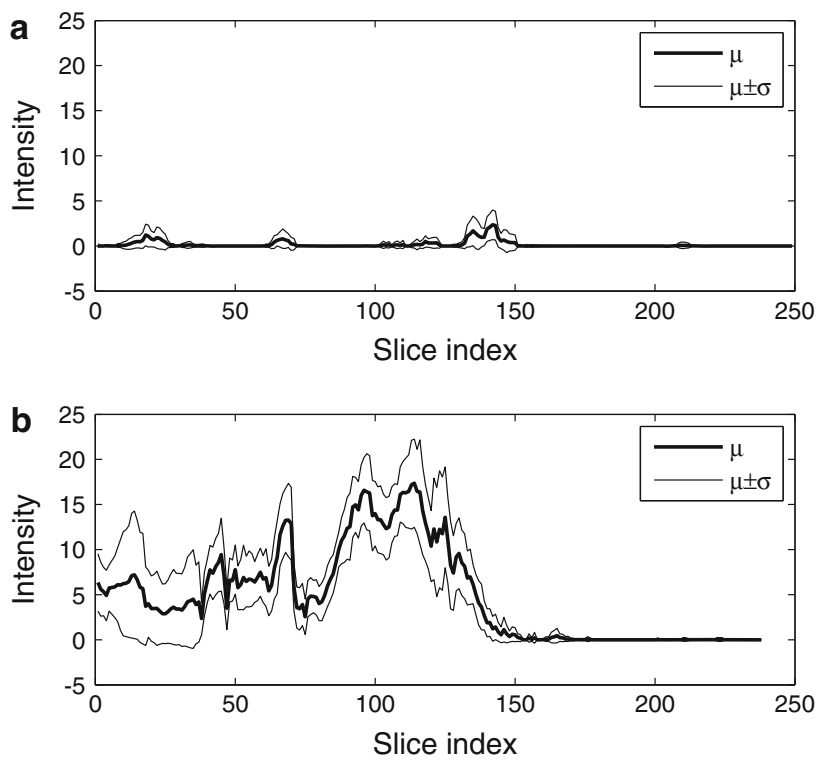

Fig. 4 The graphs show the variation of the pixel intensity in $1 \times 1 \mathrm{~mm}^{2}$ region around the manual annotated lumen centerlines. The first graph is from a sequence which almost does not suffer from speckles inside the lumen. The second sequence shows a more difficult case

If point $O$ is located at a position where all surrounding pixels have an intensity higher than $\tau$, the algorithm fails. This situation may occur when there is a large variation of intensities along the lumen, due to local variations in the amount of speckle noise, as shown in Fig. 3. Figure 4 displays the mean pixel intensities along manually annotated centerlines in two different patients. It shows that the $\mu$ and $\sigma$ values estimated from the three initial seed points are not always representative for the entire sequence. We experimented with several standard denoising methods (e.g., bilateral filtering, median filtering), but these did not solve the problem, since it is not only the standard deviation of the noise that increases, but also the mean intensity.

If the algorithm arrives at a slice where all neighbor points of point $O$ have a value larger than $\tau$, it leads to rays with a zero length during the raycasting in Step 1. In case of such an exception, the algorithm tries to restart from the other end of the centerline. Five cases are distinguished, depending on where the exception occurs (Fig. 5). 


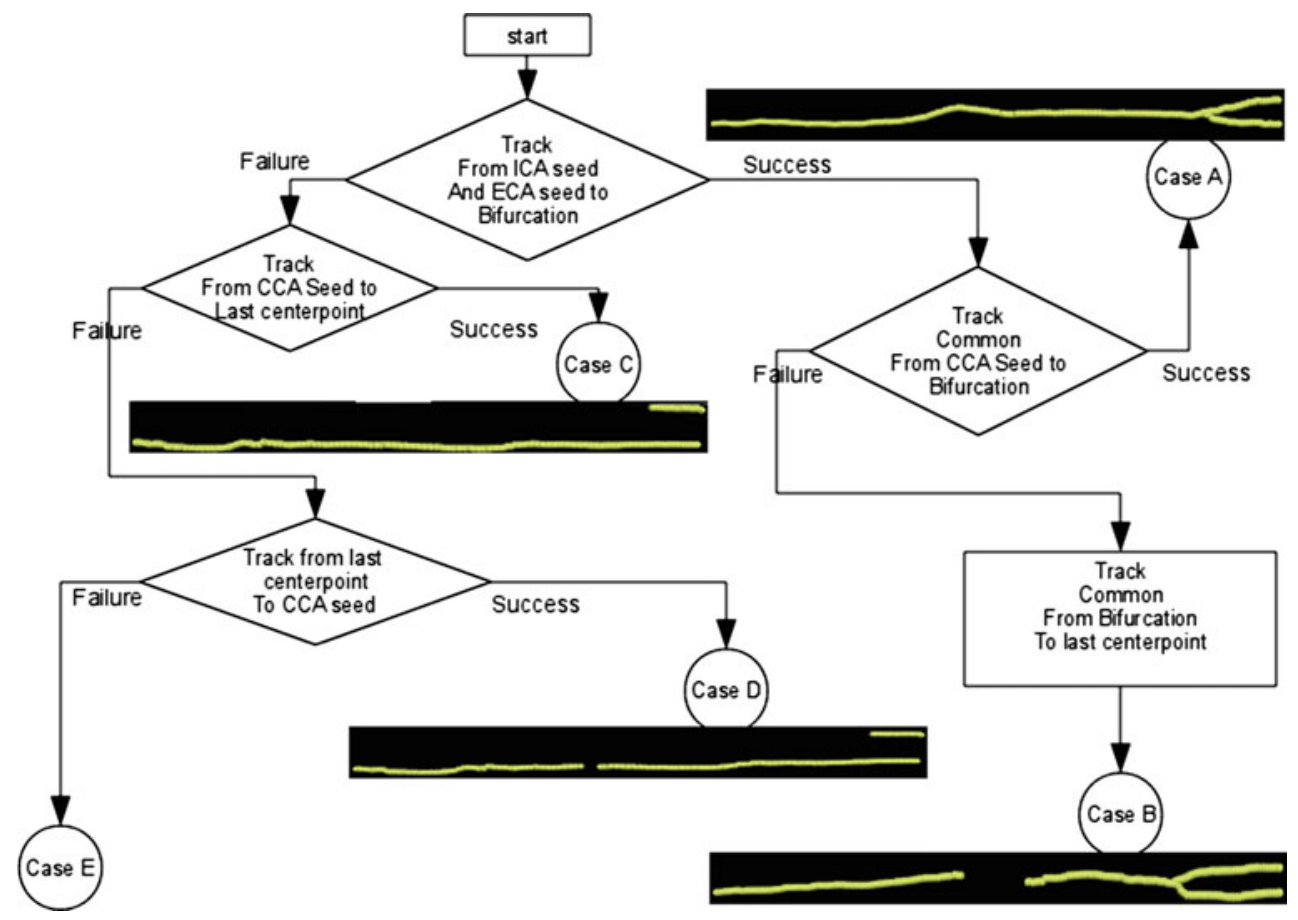

Fig. 5 Cases flow chart

The case that the algorithm succeeded in reconstructing the centerline without presenting failures in any slice is labeled Case A.

If the algorithm fails in the common carotid, the bifurcation point is used as a new seed point (Case B). Eventually the algorithm will not be able to calculate all the centerpoints from this direction either, as the region with speckles will be encountered from that side as well. The centerpoints in the intermediate slices with speckles will be defined after interpolating the calculated points with the spline curve.

If the centerpoint cannot be estimated in a slice in the ICA or ECA, the initial seed point in the common carotid is used as a seed point and it stops when the specific slice is reached (Case C). In this case, the algorithm will not find the bifurcation, but there will be at least one landmark (centerpoint) per slice.

If case $\mathrm{C}$ occurs, but the centerline cannot be estimated completely in the CCA either, the last centerpoint of the vessel in which the centerline was successfully estimated is used as a reference (Case D); i.e., if the problem occurred in the ECA, the last centerpoint in the ICA is used to match the remaining path of the CCA.

If in the last step of case $\mathrm{D}$, the centerline cannot be estimated again, the algorithm stops (Case E).

The algorithm was fully written in $\mathrm{C}++$, the code is compliant with the GCC compiler and it was stored in a static library. The library was loaded in a MeVisLab (http://www. mevislab.de/) module. In the MeVisLab environment, the
GUI interface was handled. At the end of the process, the shape of the centerline in 3D can be observed with the help of rendering tools (Fig. 6).

\section{Experiments}

Motion tracking and 3D centerline construction

We attached a magnetic tracking sensor, Flock of Birds (FOB), to the US probe to register the motion during the free-hand acquisition. The sensor was attached using a custom-made tool. The FOB sensor provides the difference in orientation and position between its own position in relation to a transmitter. Since the transmitter remains fixed during the acquisition, the sensor movements allow us to track the probe displacement.

We used the Stradwin software to register the spatial coordinates with a specific image. It gathers the images of a video stream provided by a video grabber that is connected to the video output of the US machine. In the end of the scanning, the information is stored in two different files, the first containing the raw data of each acquired image and the second the information related to the position sensor and the whole software calibration. The transformation to world coordinates can be read from these files, and applied to the $2 \mathrm{D}$ centroids to obtain a 3D centerline. Figure 6 shows an example performed on data gathered from a volunteer. 


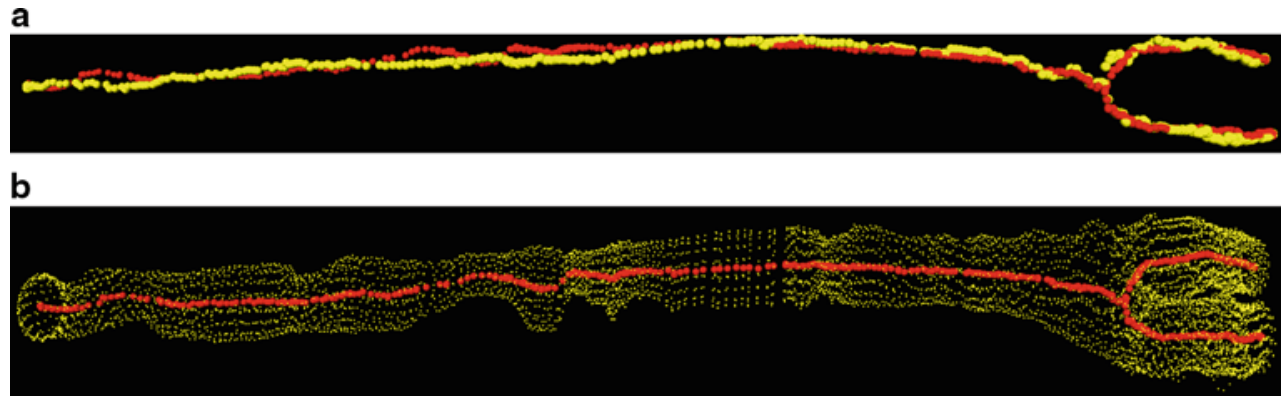

Fig. 6 Representation of the carotid artery's center points after the transformation to 3D coordinate system using the Stradwin information. The first image shows the manual annotation (yellow) and the results obtained with the algorithm (red). The second image shows a point cloud representing the borders of the ellipses calculated after the step 6 . Note that it was not our aim to accurately segment the carotid artery. Our aim is to extract the lumen centerline in a robust and efficient way

Table 1 Classification of the examinations from 1 to 19

\begin{tabular}{|c|c|c|c|c|c|c|c|c|c|c|c|c|c|c|c|c|c|c|c|}
\hline Exam & 1 & 2 & 3 & 4 & 5 & 6 & 7 & 8 & 9 & 10 & 11 & 12 & 13 & 14 & 15 & 16 & 17 & 18 & 19 \\
\hline A & $\mathrm{x}$ & $\mathrm{x}$ & & & $\mathrm{x}$ & $\mathrm{x}$ & & $\mathrm{x}$ & $\mathrm{x}$ & & & $\mathrm{x}$ & & $\mathrm{x}$ & $\mathrm{x}$ & & $\mathrm{x}$ & $\mathrm{x}$ & $\mathrm{x}$ \\
\hline B & & & $\mathrm{x}$ & & & & & & & & $\mathrm{x}$ & & $\mathrm{x}$ & $\mathrm{x}$ & & $\mathrm{x}$ & & & \\
\hline $\mathrm{C}$ & $\mathrm{x}$ & $\mathrm{x}$ & $\mathrm{x}$ & $\mathrm{x}$ & $\mathrm{x}$ & $\mathrm{x}$ & $\mathrm{x}$ & $\mathrm{x}$ & $\mathrm{x}$ & $\mathrm{x}$ & & $\mathrm{x}$ & $\mathrm{x}$ & $\mathrm{x}$ & $\mathrm{x}$ & $\mathrm{x}$ & $\mathrm{x}$ & & \\
\hline D & $\mathrm{L}$ & $\mathrm{R}$ & $\mathrm{L}$ & $\mathrm{L}$ & $\mathrm{R}$ & $\mathrm{L}$ & $\mathrm{L}$ & $\mathrm{L}$ & $\mathrm{L}$ & $\mathrm{L}$ & $\mathrm{R}$ & $\mathrm{L}$ & $\mathrm{L}$ & $\mathrm{L}$ & $\mathrm{L}$ & $\mathrm{R}$ & $\mathrm{L}$ & $\mathrm{R}$ & $\mathrm{R}$ \\
\hline $\mathrm{E}$ & $\mathrm{x}$ & $\mathrm{x}$ & $\mathrm{x}$ & $\mathrm{x}$ & $\mathrm{x}$ & $\mathrm{x}$ & $\mathrm{x}$ & $\mathrm{x}$ & $\mathrm{x}$ & & $\mathrm{x}$ & $\mathrm{x}$ & $\mathrm{x}$ & $\mathrm{x}$ & $\mathrm{x}$ & $\mathrm{x}$ & $\mathrm{x}$ & & \\
\hline
\end{tabular}

Rows: $A$ mild speckles in at least one slice, $B$ speckles in the whole lumen in at least one slice, $C$ missing borders, $D$ the side of the carotid: L-left or R-right, $E$ presence of plaque

Evaluation on patient data

\section{Subjects}

We evaluated the method by comparing the centerpoints estimated by the algorithm with centerpoints estimated by human manual annotation in every single slice. Nineteen different clinical examinations from 15 patients were tested. The exams 1 and 2 belong to one patient, exams 4 and 5 belong to a second patient, and exams 17, 18, and 19 belong to a third patient. The remaining examinations are from different patients. All the examinations were performed with a Philips iU22 system, using the Philips L9-3 probe. The pixel spacings were in a range between $0.064 \times 0.064$ and $0.095 \times 0.095 \mathrm{~mm}^{2}$. Number of slices in each sequence varied from 215 to 249 .

The FOB tracking system was not yet used in these studies, since we were aiming to evaluate the results of the algorithm taking in consideration only the issues related to the ultrasound image, such as missing borders and sensitivity to noise. A subjective quality examination was performed on all images classifying the images according to certain characteristics. The result of this examination is shown in Table 1 .

\section{Algorithm evaluation}

Figure 7 shows box plots of the Euclidean distances between the manual and calculated centerpoints for each examination.
In cases where the algorithm could not find the bifurcation due to speckles, the slices where no centerpoints were found in the ECA or in the ICA were not compared. This happened in exams 13,14, and in exam 16 using the second set of seed points. The average Euclidean distance between manual annotations and the algorithm results among all the examinations was $0.82 \mathrm{~mm}$. For each examination, the percentage of these Euclidean distances below $2 \mathrm{~mm}$ was calculated; the average over all examinations was $92 \%$. In this experiment, the parameters $\sigma_{\min }$ and $\rho$ were manually tuned by checking visually the results with different values. The best results were achieved with $\sigma_{\min }=4$ and $\rho=30 \%$.

In every measurement, the manual and calculated markers indicated a different position of the beginning of the bifurcation. The algorithm defines the bifurcation as the point where the internal and external centerpoints are less than $1 \mathrm{~mm}$ away from each other. In these specific slices, the closer of the two centerpoints is chosen to be compared with the common centerpoint in the other annotation. When the algorithm finds the bifurcation in a different slice than in the manual annotation this distance can be considerable, like in exams 3,6, and 7. The centerpoint is not located outside the lumen, but it lies between the manually annotated centerpoints.

To evaluate the robustness with respect to the choice of the initial seed points, each dataset was evaluated with two different seed point sets and the results were compared 
Fig. 7 Euclidean distance of the algorithm center points and the center points chosen manually in $\mathrm{mm}$ (a). Euclidean distance of the algorithm center points in the first and second set of seed points in $\mathrm{mm}(\mathbf{b})$.

Symbol for the points that lie outside 3 times the interquartile range is ' $\mathrm{o}$ ', default symbol for points between 1.5 and 3 times the interquartile range is ' + '
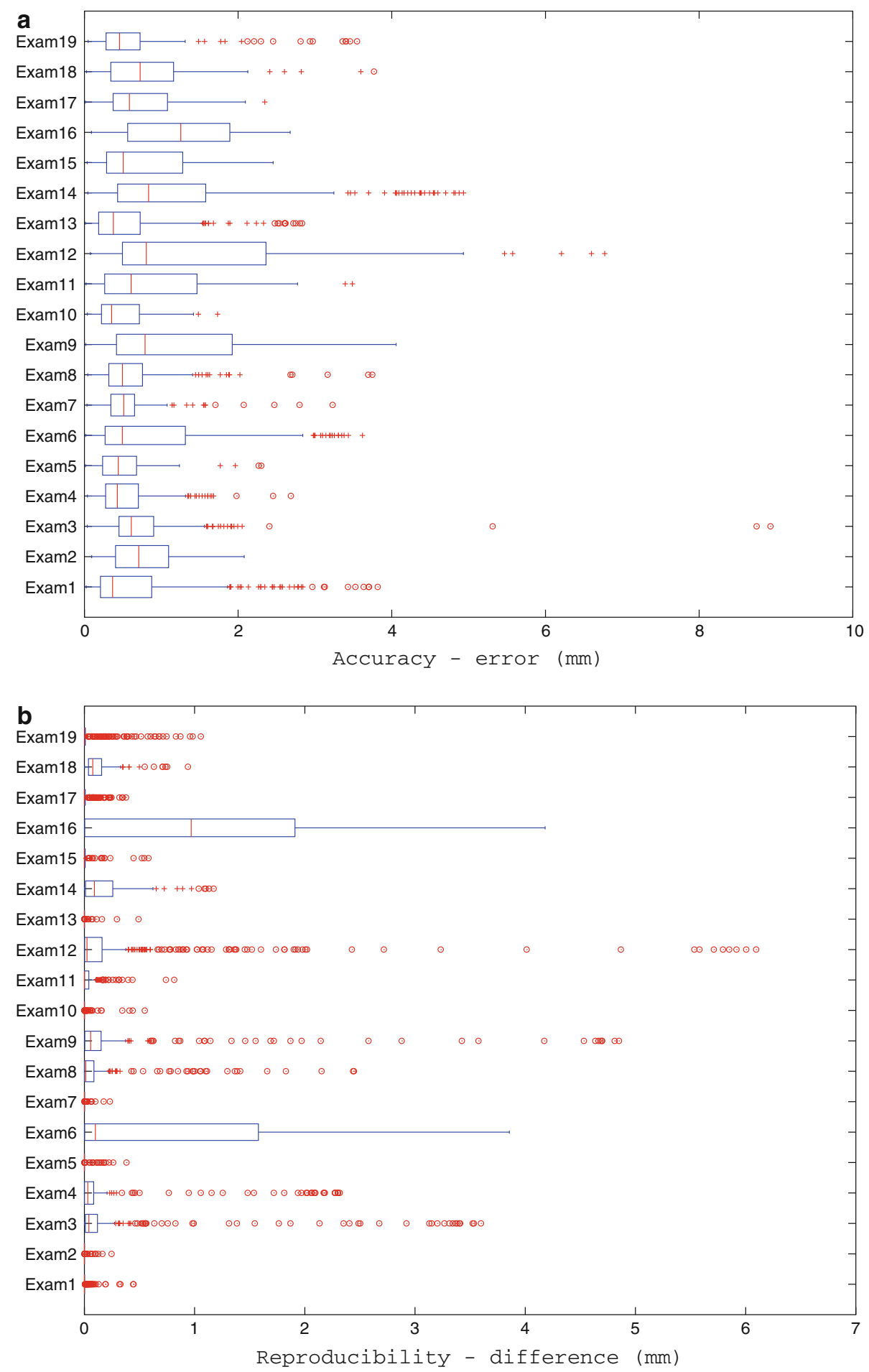

for every examination. Figure 7 shows for each examination, a box plot of the Euclidean distance between the centerpoints produced by the algorithm with two different sets of seed points. In the exam 16, the median had a value bigger than $1 \mathrm{~mm}$ because using the second set of seed points, the algorithm was not able to find the bifurcation.

\section{Parameters testing}

To demonstrate the impact of parameter values, we applied different sets of parameters and calculate the euclidean distance with the manual annotated points. On each subject, we used five different configurations of parameters sets, varying the $\sigma_{\min }$ and $\rho$. Table 2 summarizes the results. 
Table 2 The label bifurcation found indicates the number of cases (out of 19) in which the bifurcation was found (which corresponds to cases A and B in Fig. 5). The label diverged expresses the number of cases in which at least one centerpoint was marked outside the lumen

\begin{tabular}{lccc}
\hline Configuration & $\mathrm{a}\left(\sigma_{\min }=2, \rho=30 \%\right)$ & $\mathrm{b}\left(\sigma_{\min }=4, \rho=30 \%\right)$ & $\mathrm{c}\left(\sigma_{\min }=8, \rho=30 \%\right)$ \\
\hline Average mean $(\mathrm{mm})$ & 0.955 & 0.817 & 1.105 \\
Average SD $(\mathrm{mm})$ & 0.744 & 0.620 & 1.253 \\
Bifurcation found & 13 & 16 & 12 \\
Diverged & 4 & 0 & 5 \\
\hline Configuration & $\mathrm{d}\left(\sigma_{\min }=4, \rho=30 \%\right)$ & $\mathrm{b}\left(\sigma_{\min }=4, \rho=30 \%\right)$ & $\mathrm{e}\left(\sigma_{\min }=4, \rho=30 \%\right)$ \\
\hline Average mean $(\mathrm{mm})$ & 1.316 & 0.817 & 3.841 \\
Average SD $(\mathrm{mm})$ & 3.805 & 0.620 & 8.747 \\
Bifurcation found & 16 & 16 & 7 \\
Diverged & 3 & 0 & 5 \\
\hline
\end{tabular}

The execution of the algorithm with configuration 'b' resulted in centerlines with all points inside the lumen. In configuration ' $a$ ', the algorithm characterizes wrongly some noise pixels inside the lumen as the vessel wall. With configuration ' $c$ ', the real boundaries of the lumen were sometimes missed, due to a higher threshold. In configuration 'd', less outlier rays were pruned, leading to wider ellipses and, consequently, sometimes divergence. The results of configuration 'e' were affected by the exclusion of too many rays, generating ellipses that poorly represented the lumen. It should be noted that the optimal choice of $\sigma_{\mathrm{min}}$ depends on the intensity range of the US images, which all were normalized to [0-255] in our case.

The algorithm in configuration ' $b$ ' was executed again on a set of 8 new image sequences, to verify if we did not overtrain our method on the 19 datasets that were used in all other experiments (since we did not perform a leave-one-out crossvalidation). The 8 new datasets originate from 4 patients (L/R artery) and had similar characteristics as the other 19 datasets. The results were visually inspected. It was verified that in all of the 8 cases, the centerline remained inside the lumen and the bifurcation was found in 6 cases.

\section{Future work and discussion}

This paper presents a methodology to estimate the 3D lumen centerline of carotid arteries scanned with free-hand 2D US. The algorithm's main objective is to calculate the vessel's centerline even if the image does not provide full information of its boundaries due to limitations in the US examination. The algorithm is robust enough to keep the centerlines inside the vessel in the absence of visible edges in the region that represents the lumen. The sensitivity to the choice of $\sigma_{\min }$ and $\rho$ was assessed by testing five different sets of parameters. The execution time of the algorithm varied from 9 to $12 \mathrm{~s}$ depending on the number of slices and centerpoints in the examination. The whole process considering the disk access and the Gaussian smoothing took between 19 and $23 \mathrm{~s}$. All experiments were performed on an Intel(R) Xeon(R) CPU E5520 with 12 gigabytes of RAM.
The definition of the threshold for the detection of lumen boundaries is currently based on the intensity distributions around the three initial seed points. This approach may fail when the noise characteristics in the lumen change along the image series. An adaptive procedure, which updates the threshold in every scan, may be beneficial in such cases.

As a future work, we aim to use this centerline estimate to register the US scans to MRI and CTA, by matching the centerlines. Despite the initial intention of acquiring a geometrical landmark of an US acquisition to match the images to other modalities such as MRI and CTA, the extracted lumen centerline could be useful on its own right. Geometrical characteristics that might be relevant for risk assessment of plaque development can be extracted from the US-based centerline, without the need for performing an additional (expensive) MRI or CTA. According to [20], it is possible to characterize the curvature, torsion, and tortuosity by applying classical differential geometry of curves on the line segments that compose the centerline. Lee et al. [21] investigated the carotid geometry and its impact in the blood flow. The exposure to the so-called "disturbed" flow may be a risk factor for atherosclerosis. Lee et al. considered that tortuosity is among the features that influence the hemodynamic disturbance. Analysis of more examinations and comparisons with centerlines extracted from other modalities will allow us to check the potential of the algorithm in identifying these biomarkers.

Acknowledgments This research was performed within the framework of CTMM, the Center for Translational Molecular Medicine (www.ctmm.nl), project PARISk (grant 01C-202), and supported by the Netherlands Heart Foundation.

Conflict of interest None.

\section{References}

1. McCarthy MJ, Loftus IM, Thompson MM, Jones L, London NJM, Bell PRF, Naylor AR, Brindle NPJ (1999) Angiogenesis and the atherosclerotic carotid plaque: an association between symptomatology and plaque morphology. J Vasc Surg 30(2):261-268

2. Aaron F, Downey DB, Cardinal HN (2001) Three-dimensional ultrasound imaging. Phys Med Biol 46(5):R67-R99 
3. Rocha R, Campilho A, Silva J, Azevedo E, Santos R (2010) Segmentation of the carotid intima-media region in B-mode ultrasound images. Image Vis Comput 28(4):614-625

4. Antiga L, Steinman DA (2004) Robust and objective decomposition and mapping of bifurcating vessels. IEEE Trans Med Imaging 23(6):704-713

5. Aylward SR, Bullitt E (2002) Initialization, noise, singularities, and scale in height ridge traversal for tubular object centerline extraction. IEEE Trans Med Imaging 21(2):61-75

6. Bouix S, Siddiqi K, Tannenbaum A (2005) Flux driven automatic centerline extraction. Med Image Anal 9(3):209-221

7. Pock T, Beichel R, Bischof H (2005) A novel robust tube detection filter for 3D centerline extraction. In: Kalviainen H, Parkkinen J, Kaarna A (eds) Image analysis. Lecture notes in computer science, vol 3540. Springer, Heidelberg, Berlin, pp 481-490

8. Santamaría-Pang A, Colbert C, Saggau P, Kakadiaris I (2007) Automatic centerline extraction of irregular tubular structures using probability volumes from multiphoton imaging. In: Ayache N, Ourselin S, Maeder A (eds). Medical image computing and computer-assisted intervention-MICCAI 2007. Lecture notes in computer science, volume 4792. Springer, Heidelberg, Berlin, pp 486-494

9. Wan M, Liang Z, Ke Q, Hong L, Bitter I, Kaufman A (2002) Automatic centerline extraction for virtual colonoscopy. IEEE Trans Med Imaging 21(12):1450-1460

10. Schaap M, Metz CT, van Walsum $T$, van der Giessen AG, Weustink AC, Mollet NRA, Bauer C, Bogunović H, Castro C, Deng X, Dikici E, O’Donnell T, Frenay M, Friman O, Hernández Hoyos M, Kitslaar PH, Krissian K, Kühnel C, Luengo-Oroz MA, Orkisz M, Smedby Ö, Styner M, Szymczak A, Tek H, Wang C, Warfield SK, Zambal S, Zhang Y, Krestin GP, Niessen WJ (2009) Standardized evaluation methodology and reference database for evaluating coronary artery centerline extraction algorithms. Med Image Anal 13(5):701-714

11. Hameeteman $\mathrm{K}$, Zuluaga MA, Freiman M, Joskowicz L, Cuisenaire O, Flórez Valencia L, Gülsün MA, Krissian K, Mille J, Wong WCK, Orkisz M, Tek H, Hernández Hoyos M, Benmansour F, Chung ACS, Rozie S, van Gils MJ, van den Borne L, Sosna J, Berman P, Cohen N, Douek PC, Sánchez I, Aissat M, Schaap M, Metz CT, Krestin GP, van der Lugt A, Niessen WJ, van Walsum T (2011) Evaluation framework for carotid bifurcation lumen segmentation and stenosis grading. Med Image Anal 15(4): 477 488. doi:10.1016/j.media.2011.02.004

12. Noble JA, Boukerroui D (2006) Ultrasound image segmentation: a survey. IEEE Trans Med Imaging 25(8)987-1010. 0278-0062

13. Abolmaesumi P, Sirouspour MR (2004) An Interacting Multiple Model Probabilistic Data Association Filter for Cavity Boundary Extraction From Ultrasound Images. IEEE Trans Med Imaging 23(6)772-784. 0278-0062

14. Golemati S, Stoitsis J, Sifakis EG, Balkizas T, Nikita KS (2007) Using the Hough transform to segment ultrasound images of longitudinal and transverse sections of the carotid artery. Ultrasound Med biol 33(12):1918-1932

15. Hammer S, Jeays A, Allan PL, Hose R, Barber D, Easson WJ, Hoskins PR (2009) Acquisition of 3-D arterial geometries and integration with computational fluid dynamics. Ultrasound Med biol 35(12):2069-2083

16. Wang DC, Klatzky R, Wu B, Weller G, Sampson AR, Stetten GD (2009) Fully automated common carotid artery and internal jugular vein identification and tracking using B-mode ultrasound. IEEE Trans Bio-med Eng 56(6):1691-1699

17. Wang D, Klatzky R, Amesur N, Stetten G (2006) Carotid artery and jugular vein tracking and differentiation using spatiotemporal analysis. In: Larsen R, Nielsen M, Sporring J (eds) Medical image computing and computer-assisted intervention MICCAI 2006. Lecture notes in computer science, vol 4190, chapter 80. Springer, Heidelberg, Berlin, pp 654-661

18. Gee A, Prager R, Treece G, Cash C, Berman L (2004) Processing and visualizing three-dimensional ultrasound data. Br J Radiol 77(suppl 2):S186-S193

19. Bradski GR Pisarevsky V (2000) Intel's computer vision library: applications in calibration, stereo, segmentation, tracking, gesture, face and object recognition. In: IEEE Computer society conference on computer vision and pattern recognition, vol 2, pp 796-797. Art no. 854964. http://ieeexplore.ieee.org/xpl/freeabs_all.jsp?

20. Piccinelli M, Veneziani A, Steinman DA, Remuzzi A, Antiga L (2009) A framework for geometric analysis of vascular structures: application to cerebral aneurysms. IEEE Trans Med Imaging 28(8):1141-1155

21. Lee SW, Antiga L, Spence JD, Steinman DA (2008) Geometry of the carotid bifurcation predicts its exposure to disturbed flow. Stroke: J Cereb Circ 39(8):2341-2347 